\title{
An Introduction to Thesis Preparing Algorithm Using MS Word 2013
}

\author{
Mohammad Nikafrooz ${ }^{1}$, Masoume Nikan², Soheila Nikakhlagh ${ }^{3}$ and Nader Saki ${ }^{3 *}$ \\ ${ }^{1}$ MA in TEFL, Ahvaz Branch, Payam Noor University, Ahvaz, Iran. \\ ${ }^{2}$ BA in Software Engineering, Khuzestan Cochlear Implant center, Ahvaz, Iran. \\ ${ }^{3}$ Associated professor of Otolaryngology, Hearing \& Speech Research Center, \\ Ahvaz Jundishapur University of Medical Sciences, Ahvaz, Iran.
}

DOI: http://dx.doi.org/10.13005/bbra/1950

(Received: 26 July 2015; accepted: 01 September 2015)

\begin{abstract}
The present study aims to introduce a series of procedures toward preparing any scientific text using MS Word software. Some journals and academic centers, based on their scientific domain, have their own styles and formatting. One of the challenges that authors may encounter while preparing any research report is to apply those formatting style to their thesis document file. At first, the paper provides some information about styles and then some information about the author's needs and problems during preparing any research report text. Finally, some new procedures are presented which enable authors to satisfy all formatting styles components within document files. We called the procedures thesis preparing algorithm. This algorithm renders some technical procedures which should be followed according to their order to achieve a desired output for thesis preparing procedures. This algorithm is based on three features: familiarity with software's tools and their functions, the order of processes, and special techniques. We believe using this algorithm, authors will gain a better performance in preparing any dissertation and research reports.
\end{abstract}

Key words: Research Report, Writing Procedures, MS Office Word, Dissertation preparation Algorithm.

In order to reach unity and standard through which articles, research reports and other scientific documents published, many different kinds of scientific writing styles have been provided to present final research reports in an acceptable and uniform manner. For this reason, different kinds of styles are introduced to the researchers. One of the most comprehensive one was presented by American Psychology Association (APA). Another one is AMA which is presented by American Medical Association. In addition to the mentioned styles, there are many other styles like IEEE, Harvard and Chicago. Many

\footnotetext{
* To whom all correspondence should be addressed. Tel.: 06132224673;

E-mail: Ahvaz.ent@gmail.com
}

journals and research centers, based on their scientific domain, choose one of these standard styles. Some journals and academic centers have their own styles and formatting, but theirs are mostly or slightly similar to standard styles introduced by (APA, AMA and on).

Styles try to establish a simple set of procedures, or rules, which would codify the many components of scientific writing to increase the ease of reading comprehension. Rules of style in scientific writing encourage full disclosure of essential information and allow us to dispense with minor distractions. Style helps us express the key elements of quantitative results, choose the graphic form that will best suit our analyses, report critical details of our research protocol, and describe individuals with accuracy and respect. It removes 
the distraction of puzzling over the correct punctuation for a reference or the proper form for numbers in text ${ }^{1}$.

\section{Authors' Needs}

The authors of all scientific papers, whether university students or researchers, need to have enough knowledge about the specific styles based on which they are going to write. Among authors, university students are the largest group who are asked to prepare many different kinds of scientific essays during their academic years.

Styles and formatting have an important role in increasing access to the content and meaning of text. The importance of obeying styles and formatting rules is of significant importance so that many articles and research reports may be rejected due to lack of adherence to these styles. In this regard, all authors must have a comprehensive knowledge about writing and formatting style so that they could be able to produce their contents according to the standards which are required by each style.

Any research article and research report typically has a standard structure. This structure helps to ease communication and interaction between reader and writer. This standard structure is known as IMRAD which stands for introduction, method, results and discussion. IMRAD is a method to make any scientific text look much more $\operatorname{logic}^{2}$. It provides many facilities to writer in expressing his or her ideas in more systematic way and also helps the reader to effectively get the main ideas and gist of the text and enables the reader to scan or skim for information in a more rapid way. IMRAD shape the skeleton and frame of research reports.

There are many books published to present the academic and scientific steps and processes in preparing any scientific texts.

Cargill and O'Connor ${ }^{3}$ in their book "Writing Scientific Research Articles: Strategy and Steps" considered the needs of those who are earlycareer researchers in the sciences. This book uses a practical approach to develop scientific skills. The book consists of three necessary stages for providing any successful text like: Developing strategy, developing story and using language. The materials provided in this book are presented in a step by step manner to help the authors prepare their manuscript scientifically.

Booth \& Colomb and Williams ${ }^{4}$ in their book known as "The Craft of Research" provided some information to meet the needs of all researchers in any field such as academic, political, or commercial. It provides the researchers with information about how to extract a research problem from a topic or a question, how to organize and provide a draft as well as how to revise a research report in order to make it more understandable and comprehensive.

There are other books like "Academic Writing and Publishing"5 and also "Guide to the Successful Thesis and Dissertation” ${ }^{\prime 6}$ which, like the above-mentioned books, suggest some techniques and trends to provide any scientific text. But none of these give information about using word processing software to prepare academic and scientific texts. There are few books which discuss procedures to type any texts by using word processing software.

Within each style, there are some formatting components which shape the content. For example, writing style of a research report may cover the following items which should be considered:
a) Page size and margin
b) paragraph indentation, spacing, alignment
c) Page numbering
d) Heading
e) Reference and so on

The above-mentioned items are some components of any writing style which authors should consider in their texts.

Scientific text files, like any other text file, need to be processed by software in order to satisfy style rules. There are many different kinds of word processing software used to prepare a text file. MS word is one of the most powerful word processing applications for creating, editing and finalizing different kinds of documents, from a one unformatted page to a full formatted thousands page $^{7}$. MS Word is full featured word processing software that enables users to create, design, and revise any document easily and professionally. It also provides tools to simply work on any shape, text, tables, images, charts and etc. within the page ${ }^{8}$. This software satisfies all authors' needs while providing any scientific content. Based on required writing and formatting styles, there are many 
different kinds of tools within this software: tools for setting up working place, formatting text and objects, inserting table of contents, references and so on.

\section{Authors' Problems}

One of the main challenges which authors encounter while providing a scientific text is how to provide their text and content in a way to be acceptable and attestable according to the standards of scientific centers. This may cause many problems which may lead to spending time and cost without obtaining desirable results. Most of problems refer to lack of enough knowledge or misusing software's tools during working with MS word. So it puts them on a long way without getting the desirable result.

The following are some problems which authors or users may encounter in the process of providing any scientific text based on writing and formatting styles while using MS Word.

Page numbering document file is one of the leading challenges which any author may encounter. Some styles are designed to have different page numberings within research report file. An amateur or maladroit author or user may find it difficult to manage this kind of page number style, so author may divide the content in three files and applies different page numbering to each separate file.

Table of content has a specific place in any systematic text, especially in books and research reports. By referring to table of contents, reader can rapidly and easily get the gist of content and its overall plan. Mostly it is observed that authors and users use nonstandard or unusual ways to provide a table of contents within their text. They allocate a separate page for table of content and write each heading and page numbers manually. But this method results in many challenges. For example, it may be necessary to revise or change the text after inserting table of content. This causes that the page numbers and headings do not correspond to each other anymore.

Text formatting is another problem which users or authors may deal with. Formatting of text consists of setting font type and size, paragraph orientation and indentation, line spacing and so on. User should first select the text then set all the required formatting items one by one. This process is very time consuming, challenging and may be boring.

There are many problems which may arise while working with objects. For example, a table or image is inserted at the middle or center of a page, but after doing some changing like changing the margins, the object may move from its original position. It requires that the user or author spend time to rearrange it again.

For encountering and preventing such problems, MS word rendered many tools in which by having enough knowledge and information about them, any user or author can provide a scientific content in right and appropriate way according to the required writing style.

\section{Thesis preparing algorithm}

In order to solve the previously mentioned problems, users and authors need to have enough knowledge in order to handle their task appropriately.

Kaelbling and Cohn ${ }^{9}$ described instructions needed to set margin, font, citation styles and figure placement by using MS Word toward designing a text based on required style for JMLR journal. They also explained how authors can use LATEX to prepare papers and suggest some recommendations toward a paper's submission processes in JMLR.

Salmani and Nadoshan ${ }^{10}$ in their book, “APA style and research report writing” tried to explain how to prepare scientific content based on APA style. They consider many important fundamental issues which are required to prepare a report based on MS word XP.

In the "Creating Research and Scientific Documents Using Microsoft Word" which was written by Mamishev and Sargent ${ }^{11}$ some technical and basic information about creating document file are suggested. The materials provided in this book are based on one of the fundamental component of MS word known as template. The authors gave some suggestions and advice in order to provide a template which covers most of proceeding authors' needs during typing. This book covered some powerful features of MS word 2013 like equations, table of content, cross references and so on. This book tries to provide a technical approach to provide a high quality and attractive document file.

The authors of this article suggest a 
scientific and practical method to help the users and authors avoid the problems which may arise during the preparation of any research report file. The processes of this method are designed based on a linear procedures in which by obeying and doing all processes one by one, all users or authors will be able to prepare any scientific research report according to any formatting and writing style. The authors called these mentioned procedures, Thesis Preparing Algorithm. Algorithm is defined as a series of actions to be done in a specific order to solve a problem. This definition composes two concepts: actions and order of actions ${ }^{12}$. Here the preparing thesis is considered as problem and procedures as actions. The authors use the word algorithm for these procedures to show that there are some actions to be done in a specific order to prepare a thesis.

\section{Thesis Preparing Algorithm features}

The mentioned algorithm is based on three features. The first feature is the familiarity with software's tools and their functions. The second is the order of processes presented in this algorithm. The last one refers to providing some special techniques which help authors to have a better performance. These three elements are the distinctive properties and attributes of the algorithm.

\section{Familiarity with Software's Tools}

MS Word is equipped with some tools which enable us to do all typing tasks successfully. But in order to have a good result in preparing a document file, it is necessary to have sufficient knowledge about these tools. Sometimes by lack of enough information about the software's tools, users will be on a wrong road which takes their time and energy without receiving the desired goal. This algorithm tries to introduce all the required tools which are existed in MS Word toward preparing scientific texts. This will assist users to get their desirable target by using appropriate tools and consuming less time and energy.

\section{Procedures Order}

Most of the time, users are faced with the conditions in which they may be forced to redo some of the previously done actions. This results from doing actions in a wrong order. Author may perform two actions in which the second one is the prerequisite for the first one. This forces the user to redo and repeat some actions especially in the case of formatting. In this algorithm, the authors suggest a uniform hierarchical order which users can follow without any worries about redoing any actions.

\section{Special Techniques}

There are some specific parts of writing style and formatting which can hardly be managed by using MS word. For example, users tend to provide the research report with an automatic table of figures supported by automatic captioning. But it requires changing the format of page numbering in order to all page number be represented with sections' numbers. This algorithm is provided with some technical trends which enable users to deal with such conditions.

This new approach provides opportunities for the users to achieve all of their goals toward preparing an acceptable research report file based on desired writing style and formatting.

\section{Algorithm Procedures}

The processes of this algorithm are based on the last version of MS word known as MS word 2013.

The mentioned algorithm is designed in following sections:

a) Managing pre-type processes

b) Preparing different kind of pages

c) Setting MS Word Styles

d) Inserting Reference

According to the above-mentioned items, the first step refers to managing pre-type processes consisting preparing software's working place and page. This is divided in two sections, working place and working page. In this section, all tasks must be done before starting to type.

\section{Setting Working Place and Page}

Before starting to work on a document it is necessary to do some settings on the file:
a) Setting software's view
b) Setting automatic file recovery
c) Setting numeral appearance
d) Setting measuring unit
e) Setting saving font

To set up a working screen view, some items like zoom, ruler, and document view must be set, so that a user can have a suitable view while working on a file. Automatic recovery provides the user with an opportunity to automatically save files in any desired time to prevent losing data by 
any accident. For numbers, users can choose how numbers can be shown, all in a specific language form, based on system setting or based on context. In measurement unit, the way that measuring units are shown can be set. In saving font section, it is taught how to save the specific fonts used within the document in order to be shown properly in any other target system without worrying about any font display abnormality. To the authors, these are essential settings which should be done before any task.

All the above-mentioned settings and processes are offered to prepare the working place for providing the users with facilities and opportunities to work in a better manner and under more appropriate conditions.

One of the basic problems which users might face is changing the place of objects and content within document file and pages. To deal with such problems, the author has offered a hierarchical process in setting the file before typing. In this section and other sections, some settings will be presented to prevent such problems. It should be noted that all the settings which are presented below must be done in the right order:

a) Page size

b) Page orientation

c) Page margin

d) Paragraph orientation

\section{Preparing Different Kinds of Pages}

As you know all research reports are composed of two main parts: primary pages and chapters. The first part is in the initial pages like acknowledgment, dedication and so on. The second part consists of pages like chapters such as introduction, literature review, method, data analysis and etc. One of the most fundamental tasks which should be done properly and technically is to prepare different pages and sections within any scientific document file like research reports. Frequently it is seen that users prepare pages manually and during their task, they encounter many problems which take a lot of time and energy and prevent them from reaching the appropriate result. These problems are problems in sectioning, page numbering, designing table of contents, setting header, and footer and so on. In this section, the technical and fundamental method to deal with such problems is presented.

\section{Setting MS word Styles}

Styles are one of the most important components of MS word which have a basic role in producing any document file. Unfortunately in a large number of books the importance of styles is ignored or is discussed shortly. But in this algorithm, styles have a specific place. Styles provide facilities in doing and formatting some parts of a document in a more proper manner. Facilities like fast formatting, inserting table of contents, and formatting objects automatically.

\section{Inserting References}

Another item which receives attention in each writing and formatting style is citation and references. There are many details within references which must be considered. These are so important that disobeying its rules may cause any scientific text to be rejected by any academic center or journal. These cause a big challenge for users and authors to consider them in each reference and citations item. In this section MS word referencing tools are discussed. By using this tool all references can be inserted and organized in different ways.

\section{CONCLUSION}

The present algorithm has various properties; familiarity with software's tools, order of processes and providing special techniques to prepare authors to have a better performance while working on scientific texts. It seems that authors, by obeying these processes in the case of a comprehensive algorithm, can achieve more success in a systematic way. It should be noted again that the presented algorithm is in the form of a linear procedure in which by obeying and doing all processes one by one, all users or authors will be able to prepare any scientific research report according to any formatting and writing style.

According to the above discussions, it is necessary to provide a comprehensive book based on the above mentioned algorithm to equip authors with some facilities to prevent possible problems or deal with oncoming challenges which may encounter during the task of preparation any scientific document file. 


\section{REFERENCES}

1. American Psychological Association. Publication manual of the American Psychological Association. New York: American Psychological Association; 2009.

2. Oermann MH, Hays JC. Writing for Publication in Nursing. New York: Springer; 2015.

3. Cargill M, O’Connor P. Writing scientific research articles: strategy and steps. Oxford: John Wiley \& Sons; 2013.

4. Booth WC, Colomb GG, Williams JM. The Craft of Research. Chicago: University of Chicago Press; 2009.

5. Hartley J. Academic writing and publishing: $a$ practical handbook. New York: Routledge; 2008.

6. Mauch J, Park N. Guide to the successful thesis and dissertation: a handbook for students and faculty. New York: CRC Press; 2003.

7. Davis GH. How to Do Everything with Microsoft Office Word 2007. New York: McGraw-Hill; 2007.

8. Shelly GB, Cashman TJ, Vermaat ME. Microsoft Office Word 2007: Complete Concepts and Techniques. Chicago: Cengage Learning; 2007.

9. Kaelbling, L.P, Cohn, D. Instructions for formatting JMLR articles: a Microsoft word template. Journal of Machine Learning Research. 2002; 3: 16-30.

10. Salmani-nodoushan M, Alavi M. APA style and research report writing. Tehran: Zabankadeh; 2004.

11. Mamishev A, Sargent M. Creating Research and Scientific Documents Using Microsoft Word. Redmond: Microsoft Press; 2014.

12. Deitel JD, Deitel MD. C++ how to Program. Boston: Prentice Hall; 2013. 